Bol. Soc. Bot. México 31: 115-137, 2 tablas, 1 mapa. 1970

\title{
ESTUDIO FLORISTICO-ECOLOGICO DE LAS ALGAS MARINAS DE LA COSTA DEL GOLFO DE TEHUANTEPEC, MEXICO*
}

\author{
por Laura Huerta M." \\ y JoRge Tirado I."
}

\section{INTRODUCCION}

Las algas marinas de la costa Pacifica de México al sur de Baja California han sido muy poco estudiadas.

En la literatura existente, los lugares de los cuales las citas son más escasas corresponden a Oaxaca y Chiapas, por lo que escogimos para el presente estudio el Golfo de Tehuantepec, considerado de Puerto Angel a Puerto Arista.

Se efectuaron 4 visitas en el año, una de primavera en mayo de 1968, otra de verano en agosto, en octubre la de otoño y en febrero de 1969 la de invierno.

Se escogieron 7 lugares para colectar en las 4 estaciones y además, en cada una de ellas, se visitaron sitios adicionales. En total se colectó en 18 localidades que aparecen en la lista florística.

La región está comprendida entre los $15^{\circ} 39^{\prime}$, de latitud $\mathrm{N}$ que corresponde a Puerto Angel, y los $16^{\circ} 26^{\prime}$, que es el punto más septentrional de la Laguna Superior; los 9350' de longitud W correspondiente a Puerto Arista, y los 96³1' que es la situación de Puerto Angel.

Desde Puerto Angel hasta Punta Chipehua la costa es rocosa con escotaduras, algunas playas de arena y acantilados y morros que son los que dominan, después hay médanos y disminuye la costa rocosa y aumentan las playas, hasta la Bahía "La Ventosa"; de ese punto hacia el oriente el litoral está formado de playas y barras de arena hasta Puerto Arista.

En esta última zona, tierra adentro, se encuentra una serie de lagunas o albuferas de gran tamaño, poca profundidad y con fondo de arena fina con materia orgánica en descomposición, que son: "Laguna Superior", que es la mayor con una longitud máxima de $30 \mathrm{Km}$ de oriente a poniente, y un

* Departamento de Botánica, Escuela Nacional de Ciencias Biológicas, Instituto Poli. técnico Nacional, México, D. F. 
ancho máximo de $19.5 \mathrm{Km}$ de norte a sur. Al sureste de ella existe otra, llamada "Laguna Inferior" que mide $31.5 \mathrm{Km}$ de noreste a suroeste, y un ancho máximo de $11.8 \mathrm{Km}$ perpendicular a la longitud. Al suroeste esta última se estrecha y comunica con otra laguna llamada "Mar Tileme", colocada de poniente a oriente que mide $21 \mathrm{Km}$ de largo, y de ancho máximo $4 \mathrm{Km}$ y de mínimo $700 \mathrm{~m}$. Al oriente de la Laguna Inferior hay otra más chica llamada "Laguna Oriental" en la que desemboca el río Ostuta. Está separada de la anterior por una península que deja un canal de comunicación entre ambas llamado Puerto Estero. Este canal está limitado en el suroeste por otra península que se forma en la continuación de la barra de San Francisco hacia el noreste, y en ella está ubicado el pueblo de San Francisco del Mar, bañado por la Laguna Oriental y un ensanchamiento de la misma en la parte suroeste al que se llama "Laguna Occidental".

El Mar Tileme está separado del mar abierto por una faja de arena larga y angosta cuyo ancho máximo es de $5 \mathrm{Km}$ y el mínimo de $1 \mathrm{Km}$ y que termina en la boca de San Francisco, la cual constituía la comunicación de estas albuferas con el Golfo de Tehuantepec. Por la escasa afluencia de agua dulce en los últimos años se cerró en 1966 la boca de Sn. Francisco, y permaneció cerrada durante 4 años, en verano de 1969 cayeron lluvias torrenciales y fuertes vientos que hicieron que se abriera de nuevo esta comunicación al mar, la cual permanece abierta hasta la fecha.

En la barra entre Tileme y el mar abierto se encuentran los poblados de San Mateo del Mar y Santa Maria del Mar. De la boca de San Francisco se continúa el litoral en forma de playa arenosa, hasta la boca de Tonalá, que es la comunicación al mar de una bahía grande y muy baja, rodeada de manglares que mide $70 \mathrm{Km}$ de largo y $12 \mathrm{Km}$ de ancho máximo y 2 Km de mínimo, llamada "Mar Muerto", la bocana mide 1,600 m de ancho y tiene 4 a $5 \mathrm{~m}$ de profundidad. Este mar sólo puede ser transitado por cayucos o lanchas. Desaguan en él muchos ríos.

A partir de la boca de Tonalá la costa continúa como playa arenosa baja y. a $13 \mathrm{Km}$ se encuentra Puerto Arista, fondeadero establecido en una playa de mar abierto.

En cuanto a las características geológicas podemos decir que: en la costa de Oaxaca predominan las rocas graníticas que se considera que deben de haberse formado en el Proterozoico y eran parte de un geosinclinal que estuvo expuesto a la erosión durante todo el Paleozoico y principios del Mesozoico. En el Jurásico pasaron a ser fondo marino y después surgieron de nuevo en épocas más recientes. 
Según los datos de la Carta Geológica de la República Mexicana (1960), las rocas de la zona de Puerto Angel se consideran como intrusivas del Paleozoico. En el litoral hacia el E-NE, continúa una región formada por rocas metamórficas del Paleozoico, hasta Salina Cruz. En la parte intermedia hay una pequeña porción del Pleistoceno, desde Salina Cruz en adelante es del Pleistoceno reciente y la región de las lagunas se pueden considerar como zona en proceso de emersión.

\section{Material y métodos}

En cada sitio se colectaron las algas de la zona litoral e infralitoral superior y se fijaron en formol al $4 \%$ para una posterior determinación en el laboratorio y el montaje y conservación en el herbario.

Se tomaron datos sobre las características del agua: temperatura, densidad, salinidad, oxígeno disuelto, por el método de Winckler; alcalinidad titulando carbonatos con $\mathrm{HCl}$ 0.IN, y utilizando como indicador fenolfaleína, y bicarbonatos con anaranjado de metilo; así como transparencia con el disco de Secchi.

Al mismo tiempo se recogieron muestras de substratos para determinar textura por el método de Bouyoucos.

Para la determinación de las algas se usaron los libros de Dawson 1952, 54, 60, 61, 62 y 63; Hollenberg 1961; Setchell 1919-20, 25 y 30, y Taylor 1945.

\section{Discusión por localidades}

Puerto Angel

Puerto Angel es una pequeña bahía con 4 ó 5 brazas de profundidad, bordeada de rocas graníticas, con dos playas de arena, en la mayor está ubicado el pueblo. Esta playa presenta declive marcado, posee fuerte oleaje y tiene un pequeño muelle, junto al cual hay unas rocas donde se encontró, en la zona de mareas, una Ulva pequeña. Un poco más abajo, en la zona infralitoral superior, en algunas rocas, se encuentran unos tapetes formados por varias algas asociadas que son: Hypnea spinella, H. pannosa, Gymnogongrus serenéii, Amphiroa, Jania adhaerens, Taenioma perpusillum, Laurencia clarionensis, Ceramium gracillimum var. byssoideum.

En total el material es escaso y difícil de muestrear por el fuerte oleaje. 
MAPA DE LA REGION ESTUDIADA

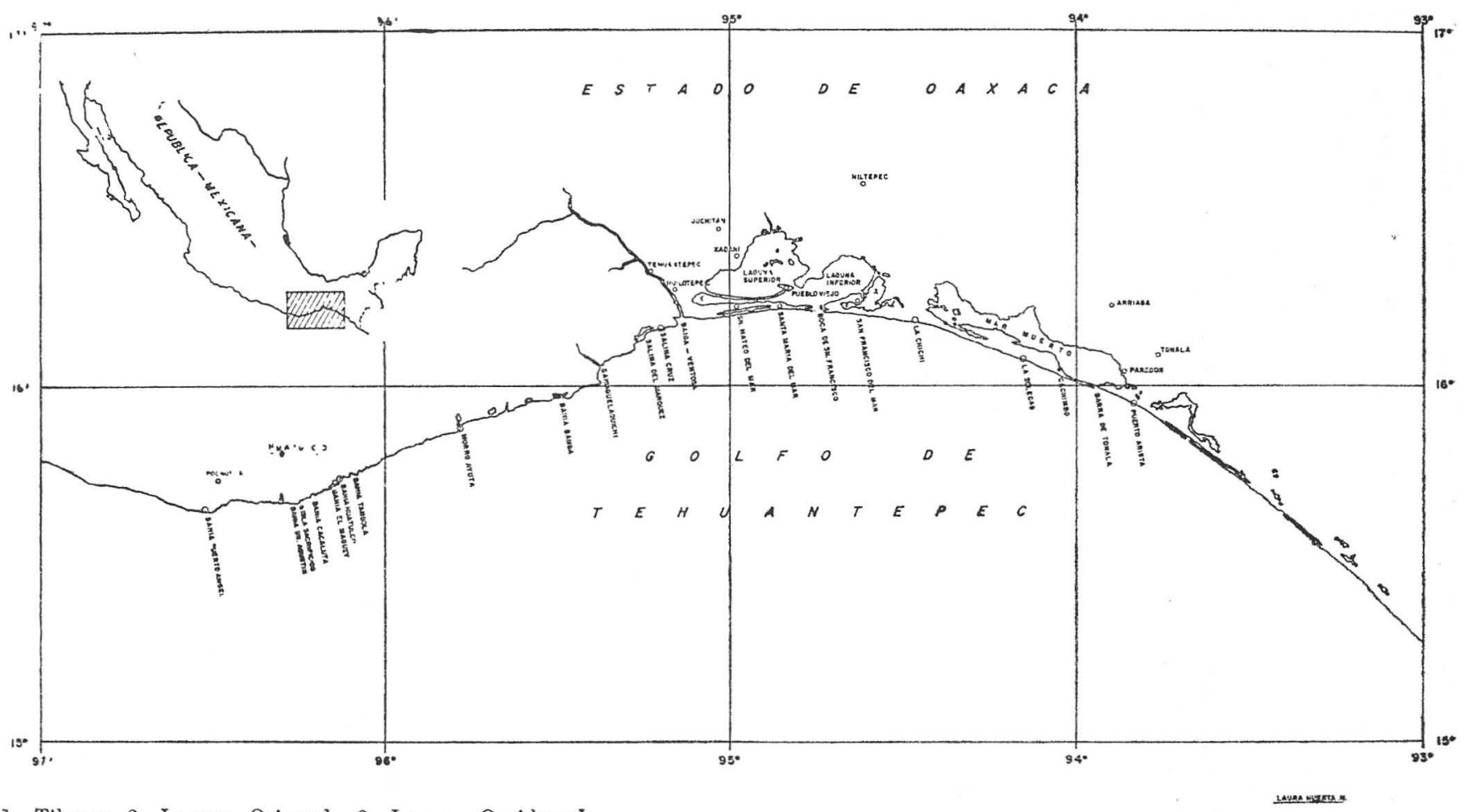

1-Tileme; 2-Laguna Oriental; 3-Laguna OccidentaI. 


\section{Isla de Sacrificios}

Frente a la bocana de la bahía "San Agustín" está la "Isla de Sacrificios", se trata de un promontorio de rocas graníticas, que no posee playas. En el lado protegido, frente a la bahía se hicieron colectas en la parte poco profunda, encontrando grandes mantos de Caulerpa sertularioides en la arena, y un poco más abajo, camas de corales.

\section{Bahia "El Maguey"}

Esta bahía posee dos playas en el fondo y una bocana muy abierta. En guijarros o conchas del piso estaban implantados algunos alcionarios con varias epibiontes, ej.: Erythrocladia pinnata, que a pesar de ser pequeña era tan abundante que el organismo que cubría parecía una rodofícea. Además se registraron Herposiphonia tenella, abundante Ceramium caudatum, unos con tetrasporas y otros con gonimoblastos, y motas pequeñas de un Ectocarpus. Sobre otros individuos había Eryihrotrichia carnea, Fosliella lejolisii, Ceramium gracillimum var. byssoideum y unos filamentos de Lynghya, etc.

\section{Bahía "Santa Cruz" o "Bahía Huatulco"}

Bahía Santa Cruz es el mejor puerto entre Acapulco y Salina Cruz.

En esta bahía la entrada está limitada por punta Manzano al norte y la punta Rosas al sur la que está rodeada de islotes. Frente a la boca está el arrecife de Piedras Blancas. En la entrada posee 20 brazas que se reducen a 5 y 3 en las orillas. En el fondo de la bahía hay una playa grande donde está ubicado el pueblo de Santa Cruz.

La Bahía presenta dirección de Noroeste en el fondo a sureste en la bocana, está bordeada de rocas graníticas que dejan lugar a unas playas arenosas pequeñas: en el lado occidental la "Yerbabuena" y "El Entrego", y en el oriental a tres playas muy pequer̃as, la más cercana a la punta la llaman "El Paraíso". Está rodeada de cerros con bosque tropical decíduo.

Esta bahía presenta lecho pedregoso, y en el fondo hacia el lado oriental posee camas de corales y en las rocas de la orilla hay grupos de alcionarios. En el centro hay además Caulerpa sertularioides. En la parte noreste se pre- 
sentan abundantes guijarros con Dicranema rosaliae. Entre los alcionarios hay Lithothamnion australe, muy abundante en agosto. En la parte sureste hay una franja de unos $30 \mathrm{~m}$ con el fondo tapizado de guijarros cubiertos de Lithophyllum y Lithothamnion laminares.

En el piso infralitoral superior se encuentran conchas cubiertas de Gelidium pusillum y Jania adhaerens y conchas de madre perla con Amphiroa magdalenensis, etc.

Además hay grandes rocas y guijarros cubiertos de unos terciopelos de pocos $\mathrm{mm}$ de alto en los que se identifican: Sphacelaria mexicana, Centroceras clavulatum, Jania pumila, Gelidiella hancockii, Herposiphonia secunda, Ceramium sp. y Polysiphonia sp.; todas ellas viven asociadas y mezcladas entre sí, o al lado unas de otras.

Un poco más profundos en las paredes de las rocas se encuentran también tapetitos formados por Hypnea pannosa, e H. spinella. En la zona infralitoral superior, casi en la parte baja de la zona de mareas, había algunos guijarros cubiertos por una pelucita verde densa y resbalosa, de unos cuantos $\mathrm{mm}$ de alto formada por Chaetomorpha natalensis forma exposita, asociada a una Enteromorpha sp. y a una Cladophora sp. muy pequeñas.

En la parte noreste de la bahía las rocas de la zona litoral presentan una coloración café claro producida por Hildenbrandia prototypus; esta coloración es más notable en invierno.

En verano el alcionario presenta varias epibiontes como Ceramium gracillimum var. byssoideum, Erythrocladia pinnata, y E. subintegra, etc.

\section{Bahía Tangola}

Tangola en zapoteca significa muy grande; en el lugar la llaman Tangolunda. En el centro de la bahía se encuentran algunos islotes y frente a su boca hay una isla llamada también Tangola.

Esta bahía posee dos playas, una mayor frente a la bocana, y otra playa chica colocada excéntricamente. La mayor parte del fondo está tapizado de corales donde se refugian pececitos en cuevas u oquedades.

Entre los corales encontramos una Ulva muy pequeña, junto con Hypnea spinella, la que llevaba un Ceramium sp. epífito, además Herposiphonia tenella y una pequeña Cladophora, Erythrotrichia carnea, Fosliella lejolisii y Enteromorpha, Gelidium pusillum y Jania adhaerens formando tapetitos que cubren las conchas. 
Caulerpa sertularioides f. longiseta forma prados y lleva entre sus rizoides una Amphiroa, una Cladophora y una cianofícea formando mota.

En algunas rocas en los lados se observaron Caulerpa sertularioides f. brevipes formando un tapiz, y entre sus rizoides había Gelidiopsis tenuis y Jania, Amphiroa, Gymnogongrus Champia parvuda, y sobre Caulerpa y mezcladas con ella Griffithsia tenuis y Geramium sp.

En algunos sitios sombreados en la parte lateral de las rocas se encontraron motas de Padina vickersiae aproximadamente a 1 ó $2 \mathrm{~m}$ de profundidad.

En otoño hay grandes rocas del piso litoral, que al bajar la marea quedan expuestas y presentan color rojo-rosa por estar cubiertas de Peyssonnelia rubra. En invierno el mar está más bajo y la zona que presentaba Peyssonnelia está seca y por debajo de esa franja, en lo que ahora es la zona de mareas, está cubierta de Hildenbrandia prototypus en forma de costras circulares o irregulares de orillas redondeadas, pero confluentes $\mathrm{y}$ las rocas se cubren por completo de color café claro rojizo.

En estas rocas hay algunos pequeños Balanus y algunas manchas verdes de una cianofícea.

\section{Morro Ayuta}

Morro Ayuta es una punta donde hay un faro. Contigua se presenta una escotadura abierta con playa arenosa que se llama Bahía Ayuta, en la que desemboca un río que lleva el mismo nombre. Ayuta significa en náhuatl "lugar de tortugas".

En la playa hay unas rocas graníticas sometidas a fuerte oleaje donde se encontró Ulva, Centroceras clavulatum y Sphacelaria. Este lugar no se pudo muestrear bien por el fuerte movimiento del agua.

\section{Bahía Bamba o Aguas Negras}

La laguna Garrapatero que es salobre y más bien representa una albufera rodeada de manglar, comunica con el mar por una barra y sólo cuando el mar está muy alto recibe agua del mismo mar. Esa bocana o barra se localiza en una escotadura de mar que en la región llaman "Aguas Negras", porque cuando hay mucha lluvia y sube el nivel de la laguna, se abre la bocana y sale agua con limo al mar prestándole esta coloración. 
La escotadura se encuentra señalada en el mapa como "Bahía Bamba", porque muy cerca hay un pueblito llamado "Rincón Bamba".

Esta escotadura es una playa abierta, recibe oleaje fuerte y en uno de sus extremos posee un morro donde se encontró una comunidad de algas formada por: Chaetomorpha media, Chnoospora pacifica, Amphiroa galapagensis, Jania mexicana, Ceramium sp., Hypnea cervicornis, Tayloriella dictyurus, Giffordia duchasaigniana, y sobre Chaetomorpha se encontró Erythrotrichia carnea.

\section{Guelaguichi}

Es una Laguna salobre con bocana cerrada, pero cuando sube el mar entra el agua hacia su interior. Está rodeada de manglar. La bocana está en una escotadura amplia que no llega a ser bahía, a la que llaman Bahía Guelaguichi. En uno de sus extremos hay un morro denominado Punta Guelaguichi, que no se pudo muestrear bien por el oleaje. Se encontraron en él: Ulva, Enteromorpha, Chaetomorpha media y Tayloriella dictyurus.

\section{Salina Cruz}

La Bahía de Salina Cruz es una escotadura entre el Cerro de Salina y cerro del Morro. Está expuesta a todos los vientos; tanto del sur, como de los nortes que soplan de noviembre a marzo.

A principios de siglo se construyó un rompeolas exterior y otro cierre interno que forman un puerto protegido que cuando está dragado permite la entrada de barcos hasta de 8 metros de profundidad.

En la actualidad este puerto es importante por poseer un oleoducto que llega desde Minatitlán. Aquí se cargan de petróleo y sus derivados buques tanque con los que se abastece la zona del Pacífico mexicano así como buques extranjeros. Además salen por este puerto maíz y otros productos agrícolas. También es importante por tener un dique seco donde se reparan barcos.

En este puerto se muestrearon dos localidades que son: la zona al pie del faro, y el rompeolas.

El cerro de Salina es de cuarcita y sobre él está construido el faro. En la base de este cerro hay una playa abierta que en su lado oriental tiene escolleras formadas por grandes bloques de cemento donde se fijan algunas algas. En la parte occidental posee rocas donde también hay algo de vegetación. 
En las escolleras en la zona de mareas, sometida a oleaje fuerte y continuo, se encuentra Chactomorpha media la que permanece durante primavera, ve. rano y otoño, pero desaparece en invierno. En otoño encontramos junto a ella Tayloriella dictyurus, sólo durante esa estación. En esta misma época se encuentran también Erythrotrichia carnea y Erytrocladia subintegra sobre Chaetomorpha.

En las rocas situadas en el lado poniente de esta playa, están presentes algunas algas formando una zonación; en la parte alta de la zona de mareas hay Enteromorpha lingulata, en seguida, hacia abajo, Hildenbrandia prototypus, después Enteromorpha micrococca y Ulva mezclada con Hypnea cer. vicornis muy pequeña, más abajo hay Padina crispata formando tapetito con Ceramium sp. y Acrochaetium sp. como epífitas. Un poco más abajo, Amphiroa mexicana, que sólo en la época en que el mar es muy bajo queda expuesta en la baja marea. En invierno la mayor parte de las algas casi desaparecen, son substituídas por Balanus, mejillones y anémonas y sólo permanecen en algunos sitios. Los Balanus se encuentran hasta la altura de la Ulva. Enteromorpha lingulata y Hildenbrandia están en malas condiciones porque es la época en que el nivel del mar es más bajo y están sometidas a una mayor sequedad en cada marea.

En una albufera ocasional formada en esta playa en otoño, se desarrolló una nata flotante de Lyngbya confervoides.

El rompeolas. En el lado poniente del rompeolas, el cual queda dentro del antepuerto, no se ve ninguna vegetación en la zona de mareas. Los barcos arrojan a veces petróleo o aceite, y las piedras se ven cubiertas de una capa obscura. De 1 metro de profundidad hacia abajo se encuentran grandes prados de Caulerpa sertularioides, con algo de Hypnea cervicornis como epífita. El agua presenta poca transparencia porque al golpear levanta la arena y hay poca visibilidad. El manto de Caulerpa aumenta en verano y en otoño, pero en invierno casi desaparece.

En esta estación baja el nivel del mar y las grandes rocas que estaban llenas de Caulerpa presentan Balanus cubriendo casi toda la superficie y la primera se conserva sólo en algunas hendiduras.

En el lado oriental del rompeolas que está expuesto al mar abierto, crece en rocas Ulva y un poco más abajo Amphiroa mexicana, la que cubre por completo algunos grandes peñascos; ambas algas viven en la zona de mareas. No se pudo muestrear bien esta localidad por el oleaje. 


\section{La Ventosa}

La Bahía La Ventosa, está formada por una playa tendida de arena que tiene en sus inmediaciones médanos de varios metros de alto. Esta bahía es poco profunda, porque en ella se depositaban los aluviones arrastrados por el río Tehuantepec. Hace algún tiempo se construyó la presa "Benito Juárez" que almacena las aguas de dicho río, y el cauce después de la presa sólo lleva una escasa corriente y la desembocadura está cerrada hace varios años.

En el borde occidental hay algunas rocas y cerca de la punta se observan cantos rodados grandes en los que había Chaetomorpha media, Enteromorpha y Ulva en la zona de mareas, de estas Chaetomorpha es la situada en la parte más alta. Más abajo hay Caulerpa sertularioides y Amphiroa mexicana. En algunos de los cantos rodados crecen Ralfsia y Lithophyllum.

A unos 3 ó 4 metros de profundidad se encontraron Padina crispata, Ulva, algo de Enteromorpha y Gelidium pusillum sobre guijarros y en conchas de ostión.

En invierno desaparecen Chaetomorpha y Entoromorpha de la zona de mareas, Ulva, está presente, pero muy pequeñita. En conchas de ostión sacadas de $3 \mathrm{~m}$ de profundidad habia Caulerpa sertularioides, Gelidium pusillum y Padina crispata, etc.

\section{Xadani}

Xadani es un pueblito de pescadores que se encuentra en el lado noroccidental de la Laguna Superior en la desembocadura del río de Los Perros. En la primavera el nivel del agua era bajo, presentando una porción desecada con fondo limoso. Había conchas de Murex que tenían implantadas motas de Enteromorpha ramulosa mezcladas con Chaetomorpha gracilis y unas ramitas de una pequeña Cladophora sp. En el suelo está diseminada la Enteromorpha compressa formando motas, las que hacen una franja en la orilla. Además, hay varias fanerógamas acuáticas. Ej.: Suaeda.

En otoño la Laguna se llena, dominando Enteromorpha.

\section{San Mateo del Mar}

Otro de los vasos o albuferas se llama Mar Tileme, en su orilla se encuentra San Mateo del Mar, pueblito de pescadores. A $2 \mathrm{Km}$ al sur de San Mateo está el mar abierto con playa tendida de arena con fuerte oleaje. 
A $1 \mathrm{Km}$ al norte del pueblo está la rivera de Tileme, que es una laguna de muy poca profundidad con playa de arena gris negruzca porque posee gran cantidad de materia orgánica en descomposición. Cuando soplan los nortes que son de otoño e invierno, salen algas dragadas por las olas, adheridas a guijarros o conchas. Aquí encontramos: Acetabularia calyculus, Enteromorpha, Dictyota divaricata, Hypnea cervicornis, Gelidium, etc.

\section{Santa Maria del Mar}

Cerca de San Mateo hacia el oriente se encuentra Santa María del Mar, en la orilla de la Laguna Inferior. Tiene el mar abierto al sur con una playa de arena carente de vegetación. Al norte del pueblo se halla la orilla de la Laguna Inferior, formada por arena con limo en la que sólo progresan algunas Enteromorpha, pero en la época de vientos arriban por el oleaje algas como por ej.: Acetabularia calyculus, Hypnea cervicornis, Dictyota divaricata, Grateloupia filicina, etc.

Al norte de Santa María del Mar, atravesando el canal está Pueblo Viejo, con una rivera cubierta por manglar, donde se encontró Enteromorpha, Hyp. nea, Lyngya y con abundancia motas de Ceramiaum affine var. peninsularis.

\section{San Francisco del Mar}

Este pueblo se encuentra en el margen de la Laguna Oriental.

Es una laguna con abundante vegetación. Está rodeada de manglar, y posee varias fanerógamas en el fondo, Ruppia marítima es la que domina, entre ella hay motas de Enteromorpha, o éstas se encuentran enredadas en las raíces de los mangles.

En los bordes de la Laguna donde no hay manglar, entre el limo hay grandes plantas de Enteromorpha intestinalis hasta de 2 metros de largo.

Muy cerca de San Francisco del Mar, en Puerto Estero, se encuentran al. gunas algas como Gracilariopsis sjoestedtii, Hypnea cervicornis, Dictyota labellata, Polysiphonia, Enteromorpha, Chaetomorpha, Cladophora, etc.

También cerca de San Francisco del Mar, en la rivera de la Laguna Inferior se encontraron Gracilariopsis sjoestedtii, Polysiphonia, Hypnea, Giffordia, etc. 


\section{Cachimbo, Oaxaca}

En la península que limita por el sur al Mar Muerto, existe una ranchería llamada Cachimbo, con manglar en sus alrededores. Aquí había Enteromorpha lingulata, Acetabularia calyculus, Gracilariopsis sjostedtii, Dictyota divaricata, Hypnea, Polysiphonia, etc.

Al sur del pueblo a unos 3 kilómetros está el mar abierto con playa de arena carente de vegetación.

\section{Paredón, Chiapas}

En la orilla del Mar Muerto, frente a la bocana está ubicado Paredón, con manglar en sus alrededores y cerca quedan las desembocaduras de dos ríos el Tiltepec y el Sanatenco. En sus cercanías hay grandes prados de Gracilariopsis sjoestedtii, enterrada en la arena, implantada en guijarros o conchas pequeñas. También se encuentra Dictyota divaricata, D. dichotoma, Polysiphonia, y algunas epífitas como Acrochaetium sp. y Eritrotrichia.

Gracilariopsis se encuentra en todo el año, pero es más abundante en otoño y en invierno disminuye su cantidad.

El Mar Muerto es muy bajo por tener declive escaso. 


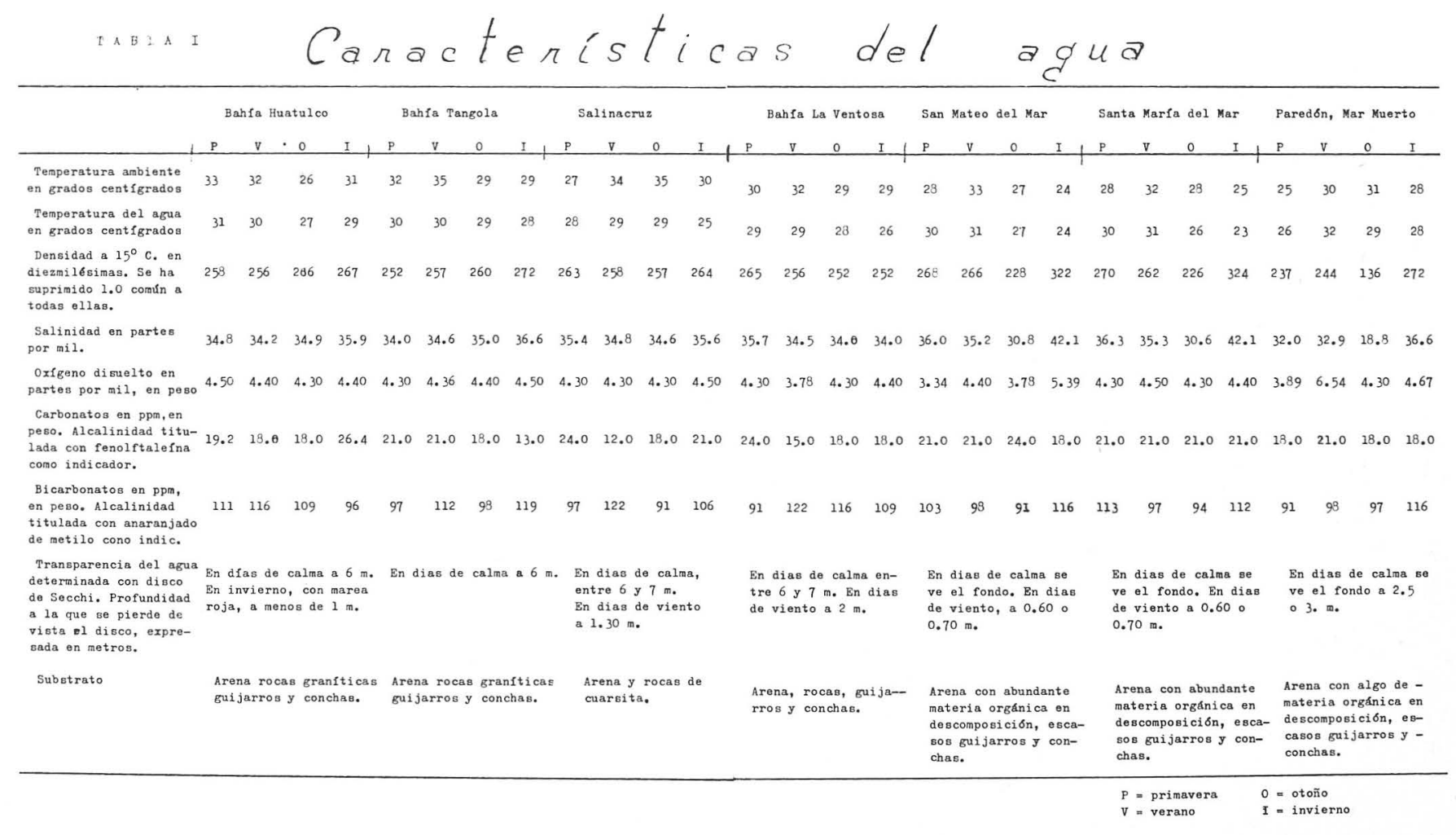




\section{Cuadro flonistico de las algas maninas de la costa del Golfo de Tehuantepec}

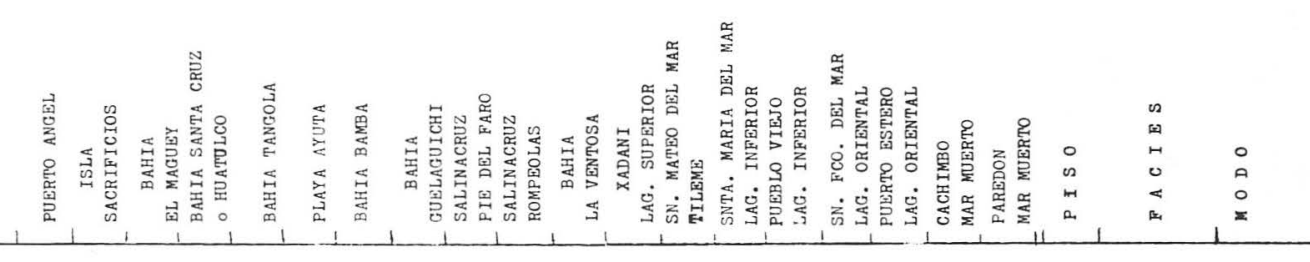

CHLOROPHY COPHYTA

Fam: Ulvacese

1 Enteromorpha acanthophora Kützing

2 E. compressa (L) Greville

3 E. fleruosa Wulfen) J. Agardh

4 E. intestinalis (L) Link

5 E. lingulaiva J. Agardh

6 E. micrococca Kutzing

7 E. marginata J. Agardh

8 E. ramulosa (J.E. Smith) Hooker

9 E. tubulosa Kqtzing

$10 \mathrm{E}$. Clathrata (Roth) J. Agardh

11 Ulva lactuca Linneeus

p

Pam: Cladophoraceae

12 Chaetomorpha antennina (Bory)Kitz.

$13 \mathrm{Ch}$. brachigona Harvey

$14 \mathrm{Ch}$. exposita (Brrgesen) Dawson

$15 \mathrm{Ch}$. Bracilis Kutzing

16 Cladophora brasiliana Martens

17 Cladophore sp.

Fam: Bryopsidaceae

18 Bryopsis pennatula J. Agardh

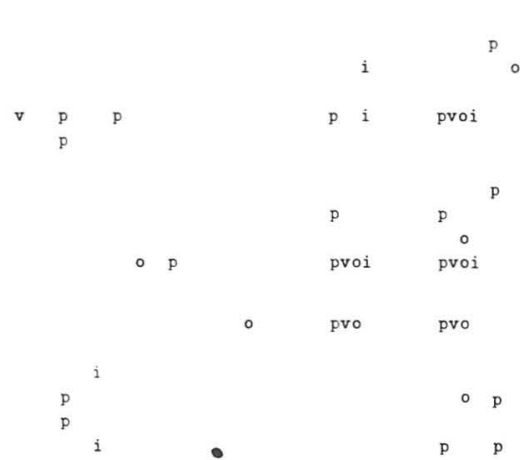

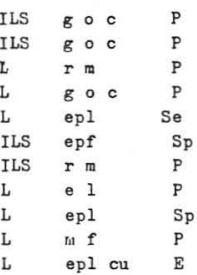

L epl cu E

ILS $\mathrm{B} 0 \mathrm{c}$

ILS epf

ILS epf

ILS $\circ \circ \quad P$ 
Fam: Dasycladaceae

19 Acetabularia calyculus Quoy et Gaimard

F. brevipes (J. Agardh) Svedelius

21 C. sertularioides F. longiseta

23 G. mitchellee (Harvey) Hamel

Fam: Ralfsiaceae

24 Ralfsia sp.

Fam. Sphacelariaceae

25 Sphacelaria mexicana Taylor

Fam: Dictyotaceae

26 Dictyota divaricata Lamouroux

27 D. dichotoma (Hudson) lamouroux

28 D. flabellata (Collins) Setchell et Gardner

29 Padina crispata Thivy

$30 \mathrm{P}$. vickersiae Hoyt

31 Padina sp.

Fam: Chnoosporaceae

32 Chnoospora pacifica J. Agardh RHODOPHY COPHYTA

Fam: Goniotrichaceae 33 Goniotrichum elegans (Chauvin)

34 Bengiopsis humphreyi (Collins)$$
\text { Hame }
$$

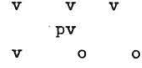

\begin{tabular}{|c|c|}
\hline ILS & $g \circ c$ \\
\hline LLS & g० $c$ \\
\hline ILS & g० C \\
\hline ILS & epl, en c \\
\hline ILS & epl \\
\hline ILS & epl \\
\hline L & epl y cu \\
\hline ILS & epf \\
\hline L & epl \\
\hline ILS & epf \\
\hline ILS & epf \\
\hline TLS & epf $\circ$ epz \\
\hline ILS & ept \\
\hline
\end{tabular}

38 E. biseriata Tanka 
Fam: Squamariaceae

42 Hildenbrandtia prototypus Nardo

43 Peyssonnelia sp.

$$
\text { Fam: Corallinaceae }
$$

44 Lithothamnion australe Foslie

45 L. fruticulosum (Kitizing) Foslie

46 L. lenormandii (Areschoug) Foslie

47 Fosliella farinose Lamouroux

48 Lithophyllum imitans Foslie

49 L. trichotomum (Heidrich) Lemoine

50 Heteroderma minutula (Foslie) Foslie

51 Amphiroa minutissima Taylor

52 A. galapaguensis Taylor

53 A. magdalenensis Dawson

54 A. mexicena Taylor

55 A. tarl

56 Jania adhaerens Lamouroux

57 J. mexicana Taylor

58 J. pumila Lamouroux

Fan: Grateloupiacea

59 Grateloupia filicina (Wulfen)C.Agardh

Fam: Gracilariaceae

60 Gracilaria verrucosa (Hudson)

$$
\text { Papenfuss }
$$

61 G. crispata Setchell et Gardner

62 Gracilaria sp. 63 Gracilariopsis sjoestedtii (Kylin)

64: Gelidiopsis tenuis Setchell et Gardner

Fam: Hypneaceae

65 Hypnea pannosa J. Agardh

66 ㅂ. spinella (C. Agardh) Kätzing

67 H. cervicornis J. Agardh

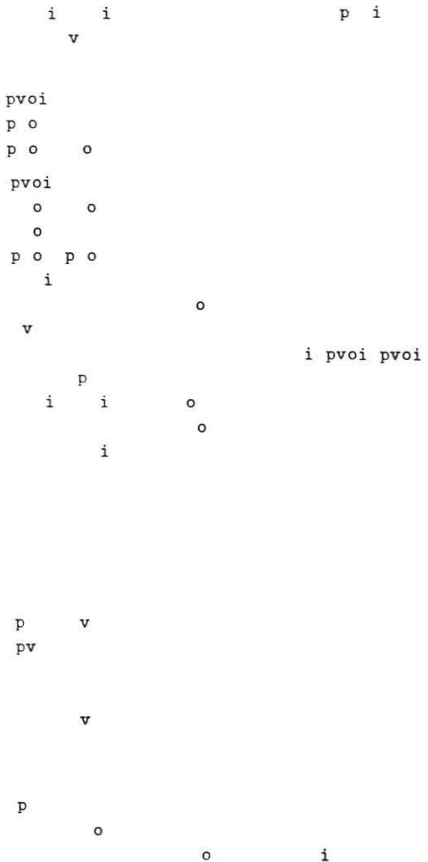

epl y cu E

ep.

ILS $\quad p s \quad s p$

ILS $\mathrm{fp} \quad \mathrm{Sp}$

ILS $f p \quad s p$

ILS $f p \quad S p$

IlS epf o epz $S p$

ILS epl $S$

L epl E

ILS en $\mathrm{c} \quad \mathrm{Sp}$

L epl y cu E

ILS epl $S$

L epl $\circ$ goc $\mathrm{E}$

$L$ epl $E$

ILS en 
Fam: Dicranemaceae

68 Dicranema rosaliae Setchell et

Gardner

Fam: Phyllophoraceae

69 Gymnogongrus serenei Dawson

Fam: Champiaceae

70 Champia parvula (C.Agardh) Harvey

Fam: Ceramiaceae

71 Griffithsia tenuis C Agardh

72 Ceramium gracilimum var. byssoideum

c. caudatum Setchell et Gardner

74 c. affine var. peninsularis (Setchell

et Gardner) Dawson

75 Ceramium sp

76 Centroceras clavulatur: C. Agardh)

Montagne

Fam: Delesseriaceae

77 Taenioma perpusillum (J. Agardh) p

J. Agardh

Fam: Rhodomelaceae

78 Polysiphonia flaccidissina Hollenberg

79 P. sonorensis Hollenbe .

80 Polysiphonia sp.

31 Herposiphonia secunda (C. Aga:dh) Ambron

32 H. tenella (C. Agardh) Ambron

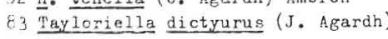
Kylin

84 Laurencia clarionensis Setchell et $p$ Gardner

25. S. subcorymbosa Dawson 


\section{RESULTADOS}

Por la naturaleza del substrato y los caracteres ambientales podemos dividir a nuestra región en 3 partes:

$1^{\circ}$ Una parte que comprende de Puerto Angel a Tangola formada por las bahías rocosas con playas de arena. Aquí están incluidos Puerto Angel, Isla Sacrificios, El Maguey, Santa Cruz y Tangola.

$2^{9}$ Otra parte rocosa de mar abierto, desde Morro Ayuta hasta Salina Cruz, pasando por Bahía Bamba y Punta Guelaguichi.

3 ? Una tercera parte formada por las lagunas o albuferas y el Mar Muerto, que son lugares bajos, con fondo de arena y limo.

En la primera el substrato es de arena con guijarros, corales muertos o conchas, donde se implantan las algas. Además estas bahías están bordeadas de rocas graníticas. Se caracteriza por presentar las siguientes especies y asociaciones: En Santa Cruz y en el Maguey el alga más conspícua es Dicranema rosaliae, que se encuentra sobre conchas o en guijarros del fondo formando prados, y a reces implantada en Lithophyllum australe.

En Santa Cruz, tanto en verano como en otoño, hay muchos Lithophyllum australe en el piso y el alcionario, que se pega a todo lo que toca, se une a él, por ser en esta época muy abundante.

Además, en verano, están presentes varias epibiontes en el alcionario, como Erythrocladia pinnata, E. subintegra, Erythrotrichia carnea, E. biseriala, Ceramium gracillimum var. byssoideum y $C$. caudatum, etc., siendo a veces muy abundantes.

Se encuentran algunas asociaciones como por ej.: un tapetito formado por Hypnea pannosa e H. spinella con Jania adhaerens, Laurencia clarionensis, Ceramium gracillimum var. byssoideum, Polysiphonia sp., Taenioma perpusillum, una Amphiroa, etc.

Hay además Ulva que lleva a Laurencia y a Polysiphonia como epífitas.

Otra asociación frecuente es una pelucita rojiza de unos $\mathrm{mm}$ de alto formada por: Sphacelaria mexicana, Jania pumila, Polysiphonia, Centroceras clavulatum, Gelidiella hancockii, Ilerposiphonia secunda, Ceramium sp., etc.

Gelidium pusillum y Jania adhaerenas se encuentran tapizando conchas y formando otra asociación.

En algunos guijarros de la parte alta del piso infralitoral superior hay una pelucita verde de unos cuantos $\mathrm{mm}$ de alto, en la que encontramos Chaeto- 
morpha natalensis forma exposita, asociada a Cladophora y a Enteromorpha muy pequeñas.

En esta misma región se observó en invierno Hildenbrandia prototypus que se encuentra tapizando grandes rocas, en las que había en verano Peyssonnelia.

También encontramos Padina, Gymnogongrus etc., solas o formando grupos.

Hay grandes prados de Caulerpa sertularioides, principalmente sobre arena o en rocas cubiertas de arena. En esta Caulerpa también hay algunas epífitas, ya sea de los remuli o de los rizoides, ej.: Hypnea cervicornis, Griffitshia tenuis, Jania, Amphiroa taylorii, Gymnogongrus, Champia, etc.

Lithothamnion es común formando masas o cubriendo guijarros o corales en el fondo de las bahías; además forma una franja sobre las rocas en la zona de mareas.

La $2^{\text {a }}$ parte de mar abierto con acantilados o morros rocosos presenta: Chaetomorpha media, Ulva muy pequeña, Enteromorpha, Amphiroa mexicana y en algunos sitios Sphacelaria y Tayloriella o Padina crispata, etc.

Chaetomorpha se encuentra en primavera, verano y otoño y desaparece en invierno.

En invierno, en la zona de mareas, se encuentran las rocas cubiertas por cirrípedos o grandes colonias de mejillones o de anémonas, que substituyen a gran parte de las algas. Tayloriella se encontró sólo en otoño.

En la parte alta del piso infralitoral superior hay abundantes prados de Caulerpa sertularioides y Amphiroa mexicana, las que a veces se encuentran también en la parte baja de la zona de mareas.

En la Bahía "La Ventosa", que es un lugar que participa de las características de Bahía Rocosa y de lugar Expuesto, encontramos flora de ambos sitios:

Chaetomorpha, Ulva, Enteromorpha de sitio expuesto, y a su vez hay conchas de ostión en 2 ó $6 \mathrm{~m}$ de profundidad, en las que encuentran Gelidium pusillum, Padina crispata, Caulerpa sertularioides, etc.

En cantos rodados de la zona de mareas hay Hildenbrandia prototipus, Ralfsia y Lithothamnion, plantas comunes en las bahías.

La tercera parte incluye a las lagunas o albuferas y el Mar Muerto, que poseen fondo de arena con mayor o menor cantidad de materia orgánica en descomposición.

En la parte noroccidental de la Laguna Superior había principalmente Enteromorpha ramulosa, E. flexuosa y E. compressa, en la orilla, formando 
motas o unidas a guijarros o a conchas de Murex, pero en Tileme, que también posee Enteromorpha en la orilla, salen dragadas por el oleaje: Acetabula. ria calyculus, Dictyota flabellata, Hypnea cervicornis, Polysiphonia, etc.

En la orilla sureste de la Laguna Inferior también hay Enteromorpha en conchas o guijarros, pero también encontramos Gracilariopsis sjoestedtii aunque escaso, Grateloupia filicina, Hypnea cervicornis, Ceramium affine var. peninsularis, Dictyota divaricata y unas motas de una asociación formada por Chatomorpha, Cladophora y Enteromorpha con una Polysiphonia, Giffordia mitchellae, Acetabularia calyculus, un Gelidium, etc.

En la Laguna Oriental abunda Enteromorpha intestinalis que llega a medir hasta dos metros o más. En esta laguna es muy abundante Ruppia maritima. En el canal de comunicación con la Laguna Inferior también hay Gracilariopsis sjoestedtii, Hypnea cervicornis, Dictyota flabellata, etc.

El Mar Muerto que, como ya dijimos, es una bahía muy grande, muy baja y rodeada de manglar, con fondo de arena y limo, posee en algunos sitios, ej. cerca de Paredón y de Cachimbo, grandes prados de Gracilariopsis sjoestedtii enterrados en el limo el cual está acompañado de Dictyota divaricata y $D$. flabellata y algunas epifitas como Polysiphonia flaccidissima; además hay algunas motas de Enteromorpha en las raíces de los mangles.

Es muy notable la abundancia del Gracilariopsis, el que llama la atención principalmente en otoño, cuando hay vientos y oleaje que lo hacen llegar a la orilla.

A estos resultados podemos agregar los que se observan en la tabla $I$.

Como vemos en la tabla sobre las características del agua, la temperatura más baja que encontramos en febrero fue de $23^{\circ} \mathrm{C}$. en Santa María del Mar, con varios días de viento norte, que produce oleaje y enfriamiento. Estos vientos son casi constantes de noviembre a marzo.

La temperatura máxima del agua fue de $35^{\circ} \mathrm{C}$. en agosto en Punta Flor cerca de Paredón, en la orilla de un manglar, en un sitio en que había Gracilariopsis sjoestedtii.

En Salina Cruz, donde tomamos las temperaturas en el pie del Faro, del lado de mar abierto, las variaciones entre verano e invierno son menores, de 4 ó 5 grados únicamente.

En cuanto a la densidad y la salinidad, podemos ver que el lugar donde se presenta menor variación es en Salina Cruz, donde los datos están tomados en mar abierto y donde no hay desembocadura cercana de ningún río.

En las bahías de Huatulco y Tangola estos factores varían un poco más, y en las albuferas o Lagunas Superior, Inferior, Tileme y Oriental compro- 
bamos que en la época de lluvias la salinidad baja a $30 \%$ y en la época seca aumenta hasta $42 \%$.

En Paredón están más acentuadas aún estas diferencias, pues junto al pueblo desembocan dos ríos: en época lluviosa baja la salinidad hasta $18 \%$ o y en época seca sube casi al doble. Los datos de Paredón probablemente no son representativos de todo el Mar Muerto, porque donde hay afluencia de agua dulce debe ser más marcada la variación que en el resto de dicho mar. Sin embargo, vemos que en Paredón disminuye la salinidad a una concentración de la mitad de lo normal en época de lluvias, y en época seca hay una salinidad más o menos igual a la del mar abierto, pues Paredón está cerca de la bocana, mientras que en las lagunas, que en realidad hace tres años que tienen la bocana cerrada y son albuferas, la salinidad en época seca es varias unidades $\%$ mayor a la del mar abierto.

Los datos sobre oxígeno disuelto en el agua de mar nos resultan un poco bajos, pues según la tabla de Fox (en Svedrup, Johnson y Flemming, 1957) para aguas con 32.5 a $36 \%$ o de salinidad y entre $25^{\circ}$ y $30^{\circ} \mathrm{C}$. los valores de oxígeno disuelto a saturación van de 5.00 a 4.46 en ppm. en peso. La mayoría de nuestros datos son menores, sin embargo son bastante próximos a los límites bajos de la tabla mencionada.

Los carbonatos, o sea el álcali titulado con fenolftaleína como indicador, varían de 12 hasta $26 \mathrm{ppm}$., pero la mayor parte de las muestras tienen 18 a 21 ppm.

Los bicarbonatos que son el álcali titulado con anaranjado de metilo como indicador, varían de 91.5 a $122 \mathrm{ppm}$. Como sabemos tanto los carbonatos como los bicarbonatos son los que regulan el $\mathrm{pH}$ del agua del mar, que se mantiene muy constante entre 7.9 a 8.1 , las mayores variaciones que se han encontrado van de 7.5 a 8.4 en casos extremos.

En cuanto a la transparencia, podemos decir que el disco de Secchi se pierde más o menos a los $6 \mathrm{~m}$ de profundidad en días de calma. En la mayor parte de los sitios obtuvimos datos menores. En Santa María del Mar y San Mateo del Mar, no llega a un metro, el disco se pierde a los 60 u 80 $\mathrm{cm}$., porque el fondo posee una alta proporción de limo y con mucha frecuencia hay vientos nortes, los que mantienen un oleaje fuerte y constante por lo que el agua tiene limo en suspensión.

En Tangola y Santa Cruz se pudo ver que el disco se pierde a 6 ó $7 \mathrm{~m}$ de profundidad.

En Santa Cruz, en febrero había algunas manchas rojas de dinoflegelados que producían turbidez, y el disco desaparecía antes de $1 \mathrm{~m}$ de profundidad. 
En Paredón sucede lo mismo que en las lagunas, y cuando sopla el norte no hay visibilidad, porque el oleaje levanta el limo y el agua se presenta revuelta. Sin embargo en los días de calma, el disco se ve perfectamente en el fondo, pues el sitio es muy bajo y las profundidades son de 1.5 a $3 \mathrm{~m}$ únicamente.

\section{CONCLUSIONES}

19. La flora marina de la Costa del Golfo de Tehuantepec es relativamente escasa, pues encontramos unas 80 especies de las que sólo unas cuantas se presentan con abundancia.

$2^{9}$ En la primera zona de las tres partes en las que dividimos nuestra región, o sea la de las "bahías rocosas"; podemos observar que en Huatulco y en el Maguey, el material recogido es más abundante, pero en Puerto Angel y en Tangola la regetación es pobre.

$3^{\circ}$ La flora de la segunda zona, que es la de rocas graníticas sometidas a fuerte oleaje es reducida y pequeña; encontramos Chaetomorpha, Ulva, Tayloriella, Jania, Chnoospora, etc., todas en el piso litoral. Esta zona es la más desértica pues hay grandes extensiones sin vegetación alguna; excepto pequeños microhabitats densamente poblados, la flora de la región es muy pobre.

4. La mayor parte de las plantas de las dos primeras zonas es de corta talla, excepto Caulerpa sertularioides, que forma prados hasta de varios metros.

5․ En la tercera zona también hay grandes extensiones desérticas forma. das por playas de arena con limo, pero hay otras partes donde la vegetación es más desarrollada, con manglares y fanerógamas acuáticas y abundantes motas de algas: en unos sitios domina Enteromorpha intestinalis y en otros Gracilariopsis sjoestedtii. De ambas hay individuos de dos metros o más, pues se presentan con abundancia y son exuberantes.

6? En cuanto a las algas dominantes en cada región podemos presentar el siguiente cuadro: 


\begin{tabular}{|c|c|c|c|}
\hline $\begin{array}{l}\text { Algas más } \\
\text { conspícuas }\end{array}$ & $\begin{array}{c}1^{a} \\
\text { Zona }\end{array}$ & $\begin{array}{c}2^{a} \\
\text { Zona }\end{array}$ & $\begin{array}{c}3^{\mathrm{a}} \\
\text { Zona }\end{array}$ \\
\hline Clorofíceas & $\begin{array}{c}\text { Caulerpa } \\
\text { sertularioides }\end{array}$ & $\begin{array}{l}\text { Chaetomorpha } \\
\text { media }\end{array}$ & $\begin{array}{c}\text { Enteromorpha } \\
\text { intestinalis }\end{array}$ \\
\hline Feofíces & $\begin{array}{c}\text { Padina } \\
\text { durvilaei }\end{array}$ & $\begin{array}{l}\text { Padina } \\
\text { crispata }\end{array}$ & $\begin{array}{c}\text { Dictyota } \\
\text { divaricata }\end{array}$ \\
\hline Rodofíceas & $\begin{array}{c}\text { Dicranema } \\
\text { rosaliae } \\
\text { Lithophyllum } \\
\text { australe }\end{array}$ & $\begin{array}{l}\text { Amphiraa } \\
\text { mexicana }\end{array}$ & $\begin{array}{c}\text { Gracilariopsis } \\
\text { sjoestedtii }\end{array}$ \\
\hline
\end{tabular}

Gracilariopsis sjoestedtii es muy abundante en verano y otoño, tanto en $\mathrm{Pa}$ redón como en Cachimbo.

A las conclusiones podemos agregar que parte de la flora encontrada, más - menos un $65 \%$, no había sido mencionada para nuestra región, es decir son localidades nuevas para la gran parte de las algas colectadas.

En la lista florística ponemos un asterisco junto a los nombres de las algas para las cuales nuestro artículo representa una nueva localidad mencionada en la literatura.

De estas $65 \%$ de las especies algunas han sido encontradas en Baja California, o se citan de Nayarit o Sinaloa; son las siguientes especies: Enteromorpha acantophora, Chaetomorpha natalensis, Giffordia duchassaigniana, $G$. mitchellae, Sphacelaria hancockii, Dictyota dichotoma, D. flabellata, Goniotrichum alsidii, Erythrocladia subintegra, Erythrotrichia biseriaia, Gelidiella hancockii, Peyssonnelia rubra, Amphiroa magdalenensis, A. taylorii, Gracilariopsis sjoestedtii, Gelidiopsis tenuis, Hypnea cervicornis, Gymnogongrus serenei, Ceramium caudatum, C. affine var peninsularis, Polysiphonia sonorensis y Laurencias clarionensis.

Otro conjunto de especies se ha encontrado en Jalisco, Colima o Guerrero, generalmente Acapulco; son las siguientes algas: Ralfsia, Padina crispata, Erythrotrichia carnea, Lithotamnion australe, Fosliella farinosa, Jania capillacea, Jania pumila, Gracilaria crispata, Hypnea spinella, Champia parvula, Griffithsia tenuis, Ceramium gracillimum var. byssoideum, Polysiphonia flac. cidissima y Herposiphonia tenella. 
Las siguientes especies no habían sido mencionadas para la costa Pacífica mexicana: Enteromorpha micrococca, E. flexuosa, E. marginata, E. ramulosa, Chaetomorpha gracilis, Ch. brachygona, Cladophora brasiliana, Acetabularia calyculus, Padina vickersiae, Bangiopsis humphreyi, Erythrocladia pinnata, Amphiroa minutissima, $y$ A. galapagensis. En cuanto a estas dos últimas, que se conocen de América Central y Sudamérica, puede ser que el límite norte de su distribución corresponda a Oaxaca. Para las Enteromorpha hay que revisar más literatura. Pero las más notables son: Acetabularia calyculus y Bangiopsis humphreyi, las cuales no están mencionadas para la región Pacífica al norte del Golfo de Tehuantepec, ni en América Central.

Algunas especies se consideran como cosmopolitas: Enteromorpha compressa, E. intestinalis, Ulva lactuca; Gelidium pusillum lo cita Dawson de toda la costa Pacífica mexicana desde las Islas Coronados hasta Salina Cruz, Oaxaca; lo mismo pasa con Hildenbrandia prototypus y Centroceras clavulatum las que también se mencionan para Centroamérica.

Del material que ya había sido mencionado de nuestra región parte fue clasificada por J. Agardh en 1847, procedente de una colecta que efectuó Liebmann en la Bahía San Agustín, Oaxaca. Este sitio es la localidad típica de: Chnoospora minima, Hypnea pannosa, Taenioma perpusillum y Tayloriella dictyurus; Dictyota divaricata que estaba en esta colecta había sido descrita previamente por Lamouroux.

Elmer Yale Dawson también visitó en alguna ocasión nuestras localidades y citó algunas especies de esos lugares: Chaetomorpha antennina de Salina Cruz, Caulerpa sertularioides de Tangola; Dictyota divaricata de San Agustín; Amphiroa mexicana de Salina Cruz, Jania mexicana de Bahía Tangola y Salina Cruz, y Herposiphonia secunda de Bahía Huatulco.

\section{RESUMEN}

Se presenta un estudio de la flora marina de la costa del Golfo de Tehuan. tepec, considerado de Puerto Angel a Puerto Arista.

Se hicieron 4 visitas a la región: en primavera, verano, otoño e invierno.

Por las características geográficas y ecológicas la región se puede dividir en 3 zonas: 1. Bahías rocosas; Puerto Angel, El Maguey, Santa Cruz y Tangola con una flora común. 2. Zona rocosa de mar abierto: Playa Ayuta, Bahía Bamba, Salina Cruz, tanto al pie del faro como en el rompeolas, $3^{3}$ Zona de las lagunas salobres y el Mar Muerto. Las llamadas lagunas son 
albuferas bajas de fondo limoso, con algunas orillas desérticas y otras cubiertas de manglares. El Mar Muerto es una bahía de muy poca profundidad con fondo de arena y limo y en su mayor parte bordeada de manglares.

Encontramos que la mayor parte de la flora presente en una zona, no se encuentra en las otras, es decir al cambiar los caracteres fisiográficos cambia la flora respectiva.

La región en general se puede considerar como pobre en su flora.

Se encontraron más de 80 especies, la mayoría pequeñas.

Para un $65 \%$ de las especies mencionadas nuestro trabajo representa una nueva localidad.

\section{BIBLIOGRAFIA}

Agardh, J. G. 1847. Mya alger fran Mexico. Ofvers al Kongl. Vetensk. Akad. Forhandl, 4 (1) : 5-17.

Dawson, E. Y. 1952. Marine Red Algae of, Pacific Mexico, Part 1. Bangiales-Corallinaceae subf. Corallinoideae. A. Hancock Pac. Exped. 17 (1): 1-240. 33 pls.

Ibid. 17 (2): $241-397$. 44 pls.
Intine Red Alg

_- 1960 Marine Red Algae of Pacific Mexico. Part 3. Cryptonemiales, Corallinaceae subf. Melobesioideae. Pacific Naturalist 2 (1): 1-125, 50 pls..

- 1961 Marine Red Algae of Pacific Mexico. Part 4. Gigartinales. Pacific Naturalist 2 (5-6): 191-341. 63 pls.

- 1962. Marine Red Algae of Pacific Mexicn. Part 7. Ceramiales: Ceramiaceae, Delesseriaceae. A. Hancock Pac. Exped. 26 (1): 1-207. 50 pls.

-_. 1963. Morine Red Algae of Pacific Mexico. Part 6. Rhodymeniales. Nova Hedwigia 5: 437-476. 19 pls.

- 1963 Marine Red Algae of Pacific Mexico. Parte 8. Ceramiales: Dasyaceae, Rhodomelaceae. Nova Hedwigia 6: 401-481. 46 pls.

Hernández Sánchez Mejorada, S. 1960. Carta Geológica de la República Mexicana, Escala 1:2000000. Comité de la Carta Geológica de México.

Hollenberg, G. J. 1961. (en colaboración con E. Y. Dawson). Marine Red Algae of Pacific Mexico. Part 5. The Genus Polysiphonia. Pacific Naturalist 2 (5-6): 345-376. 7 pls.

Setchell, W. A. and N. L. Gardner. 1919. The Marine Algae of the Pacific Coast of North America. Part 1. Myxophyceae. Univ. Calif. Publ. Bot. 8: 1-138. 8 pls.

- 1920. The Marine Algae of the Pacific Coast of North America. Part 2. Chlorophyceae. Ibid 8: 139-381. 25 pls.

- , 1925. The Marine Algae of the Pacific Coast of North America. Part 3. Melanophyceae. Ibid. 8: 383-898. 74 pls.

, 1930. Marine Algae of the
Calif. Acad. Sci. 19: 109-215, 15 pls.

Svenrup, H. U. M. W. Johnson and R. H. Fleming. 1957. The Oceans. Pretice-Hall, Inc. $1087 \mathrm{pp}$.

Taylor, W. R. 1945. Pacific Marine Algae of the Allan Hancock Expeditions to the Galapagos Islands. A. Hancock Pacific Exped. 12: 1-528. 100 pls. 